of CAPS in multiorgan failure form regressed 14 days after the first administration, SLEDAI score decreased to 32 points, GAPSS to 4 points. After 4 weeks complete B-cell depletion was achieved. A month after the course of rituximab she achieved remission, which lasts 4 years already. ANA and antiphospholipid antibodies weren't detected.

Patient E., 20, with primary APS, cardiolipin positivity, $\beta 2-$ glycoprotein-1 and lupus anticoagulant, thrombocytopenia, livedo reticularis; CAPS-like thrombotic microangiopathy type with damage to cerebral vessels, lung vessels, recurrent pulmonary embolism for six months, deep leg vein thrombosis. GAPSS activity before treatment was 17 points. Pulse therapy wasn't performed. Therapy rituximab $375 \mathrm{mg} / \mathrm{m}^{2} 1$ time per week, 4 weeks was administered. Multiple organ failure also regressed 10-14 days after 1 administration of rituximab. GAPSS score decreased to 10 points. Incomplete B-cell depletion was achieved.

Conclusion Thus, rituximab demonstrated high effectiveness in CAPS in both cases. Rituximab allowed to reach multiple organ failure regression and a persistent effect was achieved.

\section{P7 RIVAROXABAN MAY TRIGGER CATASTROPHIC ANTIPHOSPHOLIPID SYNDROME}

${ }^{1}$ Romain Stammler, ${ }^{1}$ Paul Legendre, ${ }^{2}$ Patrice Cacoub, ${ }^{1}$ Philippe Blanche, ${ }^{2}$ Jean Charles Piette, ${ }^{1}$ Nathalie Costedoat-Chalumeau. ${ }^{1}$ Internal Medicine Dept., AP-HP Cochin Hospital, Paris; ${ }^{2}$ Internal Medicine Dept., AP-HP La Pitié-Salpêtrière Hospital, Paris, France

\subsection{6/lupus-2020-eurolupus.56}

Background Catastrophic antiphospholipid syndrome (CAPS) is the most severe complication of antiphospholipid syndrome (APS). Vitamin K antagonists (VKAs) are the reference treatment for preventing relapsing thrombotic complications in patients with APS, but direct oral anticoagulants (DOACs), such as rivaroxaban, are nonetheless sometimes used in patients with antiphospholipid antibody profiles or APS. Recent studies showed that DOACs were associated with more arterial thromboses among patients with APS. The potential role of DOACs as a trigger factor of CAPS is not known.

Methods We describe two patients who developed a CAPS in the week following the institution of rivaroxaban.

Results We report the onset of definite CAPS in the week following introduction of rivaroxaban treatment in two patients, one with APS and the other with antiphospholipid laboratory findings only. Both were triple positive for antiphospholipid antibodies. The affected organs were the heart, kidneys, skin, and liver for Patient 1 , and the heart, kidneys, skin, adrenal gland, and central nervous system for Patient 2. The causative role of rivaroxaban is highly probable given that (1) CAPS occurred rapidly after this treatment was started, (2) an alternative trigger factor was found in Patient 1 only (a colonoscopy), and (3) Patient 1 had been clinically stable for 18 years with VKA as anticoagulant treatment, while Patient 2 did not have APS and had had no symptoms for 4 months (rivaroxaban had been introduced because at a scheduled visit, she reported neurological symptoms that occurred four months before and were retrospectively compatible with a brain transient ischemic accident). One similar case was reported in 2017, also following the introduction of rivaroxaban, in a patient with triple positive venous thrombotic APS who was stable for years on warfarin and who developed definite CAPS (involvement of the myocardium and adrenal glands as well as a pulmonary embolism) in the week after rivaroxaban 20 mg daily replaced warfarin to meet the patient's desire for a less burdensome treatment. ${ }^{1}$ Finally, in the randomized study published by Ordi-Ros et al, one of the patients treated with rivaroxaban developed a CAPS. ${ }^{2}$

Conclusions These two cases, as well as two previous reported cases, underline the importance of avoiding DOACs in patients with APS, especially those triple positive for antiphospholipid antibodies. VKAs must remain the reference anticoagulation treatment in this setting.

\section{REFERENCES}

1. Crowley MP, Cuadrado MJ, Hunt BJ. Catastrophic antiphospholipid syndrome on switching from warfarin to rivaroxaban. Thromb Res 2017; 153; 37-9.

2. Ordi-Ros J, Saez-Comet L, Pérez-Conesa M, Vidal X, Riera-Mestre A, Castro-Salomo $A$, et al. Rivaroxaban versus vitamine $K$ antagonist in antiphospholipid syndrome: a randomized noninferiority trial. doi: 10.7326/M19-0291. [Epub ahead of print]; 2019

\section{P8 REFRACTORY THROMBOCYTOPENIA IN A PATIENT WITH SYSTEMIC LUPUS ERYTHEMATOSUS AND SECONDARY ANTIPHOSPHOLIPID SYNDROME}

${ }^{1,2}$ Rita Torres, ${ }^{1}$ Maria João Gonçalves, ${ }^{1,2}$ Nuno Gonçalves, 1,2Santiago Manica ${ }^{1,2}$ Agna Neto, ${ }^{1,2}$ José Marona, 'Manuela Costa, ${ }^{1,2}$ Fernando M Pimentel-Santos, ${ }^{1,2}$ Jaime C Branco. ${ }^{1}$ Rheumatology Dept., Centro Hospitalar Lisboa Ocidental, Hospital de Egas Moniz, Lisboa; ${ }^{2}$ NOVA Medical School, Universidade NOVA de Lisboa, Lisboa, Portugal

10.1136/lupus-2020-eurolupus.57

Background Thrombocytopenia is a frequent hematological manifestation in patients with systemic lupus erythematosus (SLE), usually treated with glucocorticoids, immunosuppressants (such as azathioprine and cyclophosphamide), intravenous immunoglobulin (IVIG) or splenectomy.

Case report A 44-year-old woman diagnosed with SLE and secondary antiphospholipid antibody syndrome was admitted in our hospital, in July 2018, presenting severe renal and haematological involvement. She underwent treatment with methylprednisolone, followed by prednisolone, and mycophenolate mofetil, with no response. In August 2018, the patient started haemodialysis, human immunoglobulin (transient response) and plasmapheresis due to evidence of secondary thrombotic microangiopathy. The refractory severe thrombocytopaenia and lupus nephritis, justify two cycles of rituximab - platelet count rising lasted for five months. Seven months later, the patient presented pancytopenia and due to high hemorrhagic risk, there was a switch from warfarin to LMWH (prophylactic dosing). One month later, anti-coagulation was suspended due to spontaneous intracranial bleeding. In August 2019, the patient had an ischemic stroke of the right occipital lobe, secondary to APS, under prednisolone and hydroxychloroquine, and with platelet count of $24.000 / \mathrm{uL}$. Because of the bleeding risk, no anti-aggregation was instituted, and she started tacrolimus - the initial good response disappeared two weeks later. After consulting a rheumatology board, tacrolimus was kept and rituximab repeated, regardless of the hypogammaglobulinemia and no lymphocytes CD19+ count.

Conclusion We experienced a severe case of secondary thrombocytopenia, that was refractory to multiple therapeutic agents. A reasonable response was obtained under rituximab. Tacrolimus is kept due to the reported cases of late effect. Ischaemic stroke is a challenging condition in patients thrombocytopenic and further clinical guidance is warranted. 